\title{
Effect of Acupuncture on Severely Affected Arm-Hand Motor Function on the Chronic Recovery Phase Patients with Post-Stroke Hemiplegia: A Randomized Parallel Controlled Trial
}

\author{
Xiao-kuo $\mathrm{He}^{1,2 \dagger}$, Qian-qian Sun ${ }^{1 \dagger}$, Cheng Chen², Guo $\mathrm{Yu}^{1}$, Dan-dan Xie ${ }^{2}$, Hui-tian Zhang ${ }^{1}$ and Li-dian \\ Chen $^{1 *}$ \\ ${ }^{1}$ School of Rehabilitation Medicine, Fujian University of Traditional Chinese Medicine, Fuzhou, Fujian Province, China \\ ${ }^{2}$ Taihe Hospital (Affiliated Hospital of Fujian University of Traditional Chinese Medicine), Shiyan, Hubei Province, China \\ ${ }^{\dagger}$ These authors contributed equally to this work \\ *Corresponding author: Chen Lidian, Fujian University of Traditional Chinese Medicine, Qiuyang No.1, Minhou Shangjie, Fuzhou \\ 350122, PR China
}

\section{ARTICLE INFO}

Received: 蔧 January 21, 2020

Published: 慧 February 05, 2020

Citation: Xiao-kuo He, Qian-qian Sun, Cheng Chen, Guo Yu, Dan-dan Xie, Hui-tian Zhang, Li-dian Chen. Effect of Acupuncture on Severely Affected Arm-Hand Motor Function on the Chronic Recovery Phase Patients with Post-Stroke Hemiplegia: A Randomized Parallel Controlled Trial. Biomed J Sci \& Tech Res 25(2)-2020. BJSTR. MS.ID.004183.

Keywords: Acupuncture; Arm-Hand Motor; Function; Rehabilitation Timing Stroke; Physical Therapy; Occupational Therapy

\section{ABSTRACT}

Background and Objectives: Effect of conventional rehabilitation methods is still not satisfactory to the severely affected arm-hand motor function on the chronic recovery phase patients with post-stroke hemiplegia. Studies have demonstrated that acupuncture can improve arm-hand motor function in stroke patients. Quchi (LI11) and Waiguan (TB5) are most commonly used in the treatment of post-stroke hemiplegia. We hope to produce new therapy that combined acupuncture with modern rehabilitation therapy and identify the effects of acupuncture at Quchi and Waiguan on cortical excitability and plasticity.

Methods: This is a prospective randomized parallel controlled trial in China of 80 chronic patients on severely affected arm-hand motor function with post-stroke hemiplegia in Brunnstrom stages I-II, randomized into conventional rehabilitation training group $(n=20)$, acupuncture with training $(\mathrm{PT})$ group $(\mathrm{n}=20)$, acupuncture priority training group $(n=20)$, and acupuncture delay group $(n=20)$. On the basis of basic treatment and conventional rehabilitation training, acupuncture will be performed 30 minutes at Quchi and Waiguan at the same time, before and after training (PT), once a day for 30 minutes, for 6 weeks ( 5 days/week). Arm-hand motor function before and 2-, 4-, 6- week after intervention was measured using the Fugl-Meyer assessment, Wolf Motor Function Test, and Brunnstrom stage classification serve as the outcome measure. Patients will undergo follow-up assessments for 4-,12- week after the intervention.

Result: The acupuncture priority training group significantly improved Fugl-Meyer assessment flexor synergy $(2.5 \pm 2.7, \mathrm{P}<0.05)$, proximal upper extremity $(4.8 \pm 5.0, \mathrm{P}<0.05)$ and Brunnstrom stages classification $(0.64 \pm 1.22, \mathrm{P}<0.05)$ compared with other groups. There were differences at conventional rehabilitation training group and acupuncture delay group in Brunnstrom stages classification scores ( $\mathrm{P}=0.044, \mathrm{P}=0.037$, respectively) were observed. No significant changes in Wolf Motor Function Test were observed. No significant differences other groups in total or proximal upper extremity FMA, WMFT scores $(\mathrm{P}=0.363, \mathrm{P}=0.277$, respectively) were observed. The results of follow-up assessments after the intervention was significant in Brunnstrom stages classification scores at the acupuncture priority training group $(\mathrm{P}=0.047)$ for 4 - week.

Conclusion: Acupuncture as an adjuvant to conventional rehabilitation training may improve arm-hand motor function with post-stroke hemiplegia in severely impaired post-stroke patients who were in the Brunnstrom stages I-II, there are benefits to starting rehabilitation therapy (defined as PT, OT) as soon as the needle removal when the acupuncture in the affected limbs. Results of this exploratory study should be interpreted with caution. 


\section{Introduction}

Stroke is a common acute cerebrovascular disease, and two million new patients are reported in China every year [1]. One study reported that $65 \%$ of patients who received post-stroke rehabilitation had arm-hand dysfunction 6 months after stroke [2]. Effect of conventional rehabilitation methods including physical therapy (PT), occupational therapy (OT), is still not satisfactory to the severely affected arm-hand motor function on the chronic recovery phase patients with post-stroke hemiplegia. Some researchers' results suggested that acupuncture combined with rehabilitation training, such as PT, OT may be effective for treating post-stroke neurological impairment and dysfunction such as motor dysfunction, dysphagia [3]. Our previous studies confirmed acupuncture combined with MP (motor practice, abduction of the index finger) could improve motor function of the healthy subjects, there are benefits to starting rehabilitation therapy (defined as PT, OT) as soon as the needle removal when the acupuncture in the affected limbs [4-7]. The present study aimed to produce new information leading to improved timing for the combined use of acupuncture and modern rehabilitation therapy and identify the effects on severely affected arm-hand motor function on the chronic recovery phase patients with post-stroke hemiplegia.

\section{Materials and Methods}

This is a prospective randomized parallel controlled trial, the purpose is to provide an new information leading to improved timing for the combined use of acupuncture and modern rehabilitation therapy, and identify the effects of acupuncture at Quchi and Waiguan on cortical excitability and plasticity. All study protocols were approved by the institutional review boards of each facility. Full study details are provided in the online-only Data Supplement. Patients were recruited from inpatient stroke centers at 2 participating facilities. First occurrence of cerebral hemorrhage and cerebral infarction course of disease ranging from 4 weeks to 1 year, aged 30-80 years old, male or female, and exhibited Brunnstrom stage I to II movement. Full inclusion/ exclusion criteria are included in the online-only Data Supplement. Informed consent signed by the patient or their family. Patients who meet the criteria will be accepted as study participants, and will be randomized into study groups by a professional statistician (age, sex, height body weight, Body mass index, course of disease, previous history of disease, Mini-Mental State Examination score, Brunnstorm stage classification, history of allergy). The both hospitals will be coded. Random numbers will be produced using the random function UNIFORM(n) in SAS 9.0 (SAS Institute Inc., Cary, NC, USA) and sorted from smallest to largest. The rank number will be chosen as the basis for selecting the corresponding coding segments of each hospital. Similarly, four groups of random numbers will be produced. Patients and assessors will be blinded to group information. On the basis of basic treatment and conventional rehabilitation training. Arm-hand motor extremity impairment before and after intervention was measured using the Fugl-Meyer assessment, Wolf Motor Function Test, and Brunnstrom stage classification.

On the basis of basic therapy (conventional medical treatment and health education), conventional rehabilitation training will be conducted for 30 minutes per day, 5 days per week, for 6 consecutive weeks in each group. Acupuncture with training group will be performed 30 minutes acupuncture at Quchi and Waiguan (at the region of arm-hand) at the same time PT was given, while acupuncture priority training group and acupuncture delay training group at the same acupuncture sizes will be performed 30 minutes before PT (priority) or after PT (delay), once a day for 30 minutes, for 6 weeks ( 5 days/week). Disposable sterilized Hawato needles (Suzhou Medical Appliance Factory, China) were used, measuring $0.30 \times 30 \mathrm{~mm}$ for acupuncture. Horizontal needling will be carried out until the desired sensation is produced, with a depth of $20 \mathrm{~mm}$ (Shi, 2008), twirling, lifting, or thrusting, and then maintained for 30 minutes. Participants in all groups were allowed to stop the session at any time if pain or fatigue was considered excessive.

\section{Outcomes}

Outcome measures were the FMA (AH section of the FMA contains 33 items [0-66 points] as well as flexor synergy [0-12 points] and the proximal AH [0-36 points]), the WMFT (Wolf Motor Function Test [0-5 points] ) needed to perform each of proximal UE (upper extremity) tasks [17 tasks]). Brunnstrom stage classification (grades from 1-6). The simplified Fugl-Meyer motor function score is commonly used to assess motor function in hemiplegic patients. The score is sensitive and reliable. Basic recovery: The motion score increased by $100 \%$, and the patient exhibits normal motor function. Significant improvement: The motion score increased by $92-98 \%$, and slight motor impairment is observed. Improvement: The motion score increased by $10-91 \%$, and moderate to severe motor impairment is observed. No changes: The motion score increased by $0-9 \%$, and severe motor impairment is present. Aggravation: Decreased motor scores and severe motor impairment are observed [8]. Basic recovery, significant improvement, and improvement are considered to reflect efficacious treatment. Brunnstrom stage classification: This classification system is utilized to evaluate the functional recovery of a hemiplegic limb and has grades from 1-6. A low grade represents poor limb function [9]. Arm-hand motor function before and 2-, 4-, 6- week after intervention was measured using the Fugl-Meyer assessment, Wolf Motor Function Test, and Brunnstrom stage classification serve as the outcome measure. Patients will undergo follow-up assessments for 4-,12- week after the intervention.

\section{Safety}

Adverse events refer to adverse medical events that occur after acupuncture but may not have a causal relationship with the treatment. If severe adverse events occur, investigators will 
report details to the principle investigator, ethics committee, and the sponsor within 2 hours. The original data will contain when, in what way (such as telephone, fax, or written document), and to whom the severe adverse events reported.

\section{Statistical Analysis}

Validity evaluation: Statistical analysis will be performed using SPSS 22.0 software (IBM, Armonk, NY, USA). Data will be expressed as mean \pm standard deviation. We will conduct tests of normality and homogeneity for variance. Normally distributed data with homogeneity of variance will be evaluated using $t$-tests. Non-normally distributed data with heterogeneity of variance will be evaluated using a double total $Z$ test. Ranked data will be analyzed using Ridit. Treatment effectiveness was evaluated by changes in FMA, WMFT (baseline versus post-intervention) using 1- and 2-sample $t$ tests (within- and between-group comparisons, respectively). Analysis of covariance was used to control for baseline FMA score. McNemar and 2-sample Wilcoxon tests (within- and between-group comparisons, respectively) were conducted. A $P$ value of $<0.05$ will be considered statistically significant. If adverse reactions occur, these data will be included in the statistics. Invalid data and data from participants who withdrew from the study will be included in the efficacy analysis. Results will follow the intention-to-treat principle. All statistical analyses were conducted by the first author who was blinded to treatment allocation.

\section{Result}

\section{Study Participation}

Of 817 patients screened between July 2013 and November 2017, 80 chronic patients on severely affected arm-hand motor function with post-stroke hemiplegia in Brunnstrom stages I-II randomized into conventional rehabilitation training group, acupuncture with training group, acupuncture priority training group, and acupuncture delay training group (Figure 1). Fourteen patients randomized to acupuncture with training group did not complete the study.

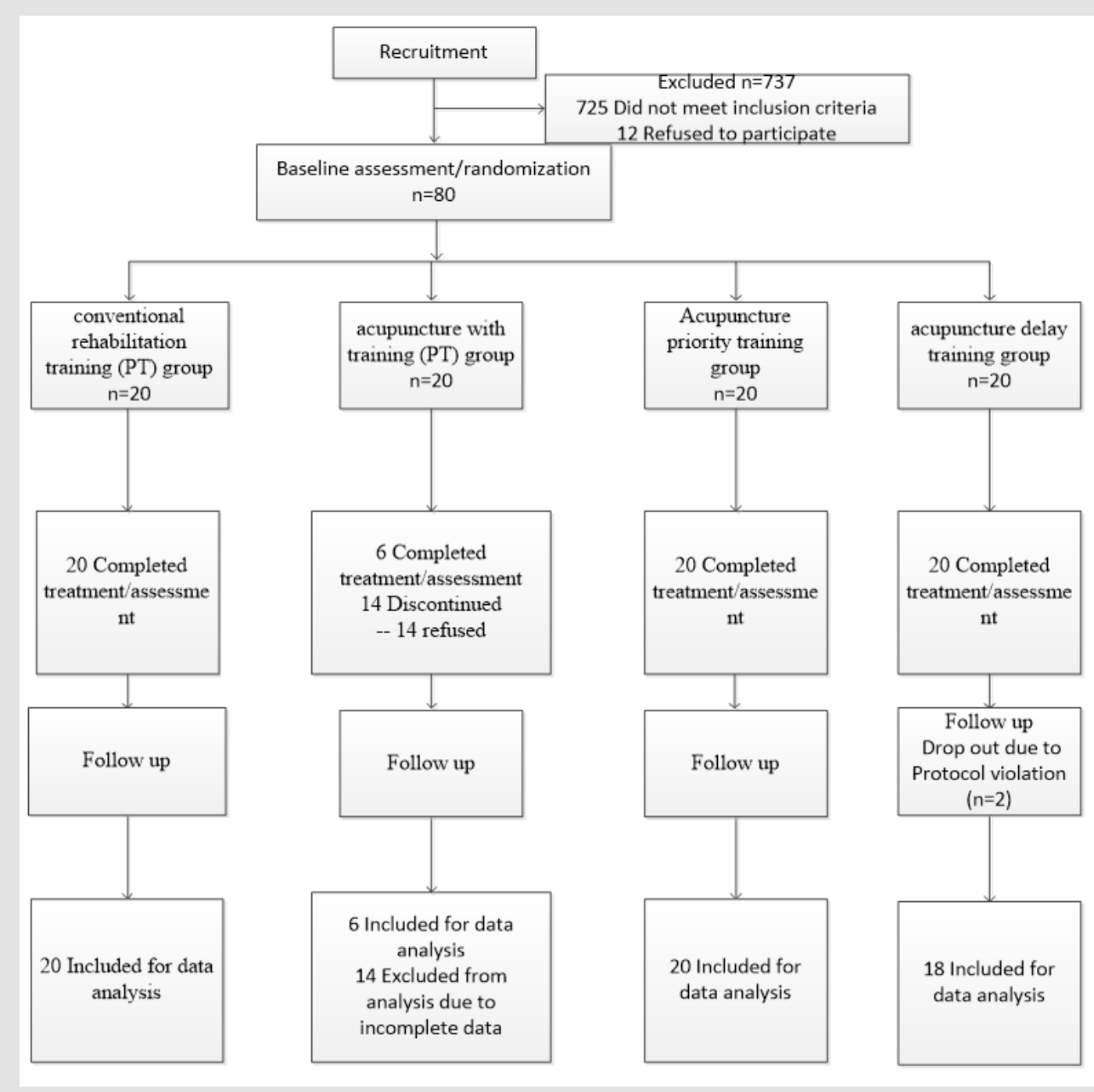

Figure 1: Consolidated Standards of Reporting Trials flow diagram of patient's recruitment and enrollment. The number of patients evaluated at each study stage. 


\section{Effectiveness}

Changes in FMA, WMFT, and Brunnstrom stage scores from baseline to the end of the intervention period between the 4 groups are reported in Table 1. Change in total FMA AH score was not significantly different between groups $(\mathrm{P}=0.245)$. However, changes in FMA flexor synergy and FMA proximal UE were different $(\mathrm{P}=0.018$ and $\mathrm{P}=0.048$, respectively). The baseline FMA, WMFT and Brunnstrom stages classification was no significantly different between groups at baseline $(\mathrm{P}=0.134, \mathrm{P}=0.211, \mathrm{P}=0.172$, respectively), the change remained significant at the acupuncture priority training group, when baseline score was included as a covariate $(1.4 ; 95 \%$ confidence interval, $0.14-2.14, \mathrm{P}=0.021 ; 1.1$; 95\% confidence interval, $0.11-2.03, \mathrm{P}=0.031 ; 1.5 ; 95 \%$ confidence interval, $0.26-3.25, \quad \mathrm{P}=0.017 ; \quad$ respectively). No significant differences other groups in total or proximal FMA, WMFT scores $(\mathrm{P}=0.363, \mathrm{P}=0.277$, respectively) were observed. There were differences at conventional rehabilitation training group and acupuncture delay group in Brunnstrom stages classification scores ( $\mathrm{P}=0.044, \mathrm{P}=0.037$, respectively) were observed. The results of follow-up assessments for 4-,12- week after the intervention were differences between the 4 groups. The change was significant in Brunnstrom stages classification scores at the acupuncture priority training group $(\mathrm{P}=0.047)$ for 4 - week, while the results of other groups are invalid $(\mathrm{P}=0.354, \mathrm{P}=0.134, \mathrm{P}=0.137$, respectively) were observed. The results of 12 - week after the intervention were no significant.

Table 1: Changes in Outcomes Four Groups from Baseline to End of the Intervention Period.

\begin{tabular}{|c|c|c|c|c|c|c|c|}
\hline \multicolumn{2}{|c|}{$\begin{array}{l}\text { Conventional Training Group } \\
\qquad(n=20)\end{array}$} & $\begin{array}{c}\text { Acupuncture with } \\
\text { Training Group }(n=20)\end{array}$ & $\begin{array}{l}\text { Acupuncture Priority } \\
\text { Group }(n=20)\end{array}$ & $\begin{array}{l}\text { Acupuncture Delay } \\
\text { Group }(n=20)\end{array}$ & Difference & $95 \% \mathrm{CI}$ & P Value \\
\hline Total FMA AH & $8.5 \pm 7.1$ & $6.9 \pm 8.8$ & $9.5 \pm 7.9$ & $7.9 \pm 7.8$ & 2.7 & -1.7 to 7.5 & 0.245 \\
\hline Flexor Synergy & $2.2 \pm 2.5$ & $1.7 \pm 5.3$ & $2.5 \pm 2.7$ & $2.1 \pm 2.4$ & 2.3 & 0.7 to 3.4 & 0.018 \\
\hline Proximal UE & $3.8 \pm 4.9$ & $-0.2 \pm 2.3$ & $4.8 \pm 5.0$ & $3.6 \pm 4.5$ & 2.9 & 0.1 to 5.4 & 0.048 \\
\hline Total WFMT AH & $-16.7 \pm 19.5$ & $-18.5 \pm 24.7$ & $-14.7 \pm 16.5$ & $-16.5 \pm 20.1$ & 1.9 & -9.1 to 11.3 & 0.674 \\
\hline Proximal UE & $-19.1 \pm 33.3$ & $-9.8 \pm 37.6$ & $-15.1 \pm 23.7$ & $-18.8 \pm 34.6$ & -8.3 & -28.3 to 9.7 & 0.431 \\
\hline Total BS & $0.46 \pm 1.02$ & $0.16 \pm 0.38$ & $0.64 \pm 1.82$ & $0.45 \pm 1.07$ & 0.01 & -0.45 to 0.49 & 0.032 \\
\hline
\end{tabular}

Note: Data are presented as mean \pm SD. CI, confidence interval; FMA, Fugl-Meyer assessment; SD, standard deviation; UE, upper extremity; WMFT, Wolf Motor Function Test; and BS, Brunnstrom Stage.

\section{Safety}

Although common post-stroke adverse events were observed at comparable frequencies in each group, no serious adverse events related to the intervention were observed.

\section{Discussion}

Acupuncture is an ancient Chinese therapeutic which has rapidly gained popularity all over the world. A number of randomized controlled trials have indicated its efficacy in the treatment of neurological disorders. Until now, it has been applied to stroke patients with motor deficits and led to a remarkable motor recovery for decades [8]. We have previously explored that electroacupuncture at acupoints and the results indicated that electroacupuncture at the acupoints, especially Quchi and Zusanli, could finally improve the recovery of motor or cognitive functions [9]. Some researchers' results suggested that acupuncture combined with rehabilitation training, including physical therapy (PT), occupational therapy (OT) may be effective for treating poststroke neurological impairment and dysfunction such as motor dysfunction [10]. A new guideline for adult stroke rehabilitation and recovery strongly suggested that there are benefits to starting rehabilitation therapy (defined as PT, OT, and SLT) as soon as the patient is ready and can tolerate it [11]. Most stroke rehabilitation protocols are based on motor learning to induce neural plasticity, which refers to the ability of the brain to develop new neuronal interconnections, acquire new functions, and compensate for impairment, that is practice-plasticity.

Some studies have used TMS to demonstrate changes in motor cortex excitability with acupuncture, and demonstrated the motor cortex excitability modulated by acupuncture under controlled experimental conditions with TMS [12-14]. These studies investigated the effect of single acupoints on the lateralized motor cortical excitability, the following question about whether these changes will be observed in the same way on stroke patients. All above studies only indicated that acupuncture could modulate the motor cortex excitability at different time (before acupuncture, the needle in situ and removal). In other words that the motor cortex excitability modulated by acupuncture whether have timedependent should be discuss. This study was the first to demonstrate that acupuncture as an adjuvant to conventional rehabilitation training may improve arm-hand motor function Chronic patients on affected with post-stroke hemiplegia in Brunnstrom stages I-II, there are benefits to starting rehabilitation therapy (defined as PT, OT) as soon as the needle removal when the acupuncture in the affected limbs. Our results have important clinical and public health implications.

The time-dependent effects of acupuncture on cortical excitability is might able to induce long-term potentiation (LTP)like plasticity and increase motor learning in human motor cortex, 
and finally could improve the motor function [15-18]. We did not use continuous or intermittent stimulation either manually or by electric means once the needle was put in situ. We cannot answer whether strengthening stimulation during the session could have yielded a positive result. Nor can we answer whether acupuncture on sites such as the ear or head could have had a different result.

\section{Conclusion}

Our findings suggest that acupuncture may be as a useful alternative to conventional rehabilitation training (such as PT, OT) as an adjuvant to therapist-guided standard rehabilitation, especially to starting rehabilitation therapy as soon as the needle removal when the acupuncture in the affected limbs, and in patients with more severe AH impairment in the Brunnstrom stages I-II. Results of this exploratory study should be interpreted with caution.

\section{Competing Interest Statement}

The author denies that he has any intention to obtain any financial interests.

\section{Acknowledgement}

None.

\section{Conflict of Interest}

No conflict of interest.

\section{References}

1. Liu R, Huang Y (2011) The management of acute stroke and post stroke in China[J]. Chinese Journal of Contemporary Neurology \& Neurosurgery 11(2): 132-133.

2. Hummel FC, Cohen LG (2006) Non-invasive brain stimulation: a new strategy to improve neurorehabilitation after stroke? [J]. Lancet Neurology 5(8): 708-712.

3. Zhang JH, Wang D, Liu M (2014) Overview of Systematic Reviews and Meta-Analyses of Acupuncture for Stroke. Neuroepidemiology 42(1): 50-58.

4. Cai Y, Zhang CS, Liu S (2017) Electroacupuncture for Poststroke Spasticity: A Systematic Review and Meta-Analysis. Archives of Physical Medicine \& Rehabilitation.

5. Cao Y, Sun N, Yang J W, Zheng Y, Zhu W, et al. (2017) Does acupuncture ameliorate motor impairment after stroke? An assessment using the CatWalk gait system. Neurochemistry International 107: 198.
6. Chen B, Tao J, Huang J (2014) The possible mechanism of electroacupuncture promoting hippocampal neural stem cells proliferation in rats with focal cerebral ischemia-reperfusion via activation of Notch signaling pathway[J]. Chinese Journal of Rehabilitation Medicine 29(5): 399-404.

7. He X, Yan T, Chen R, Ran D (2012) Acute effects of electro-acupuncture (EA) on hippocampal long-term potentiation (LTP) of perforant pathdentate gyrus granule cells synapse related to memory. Acupuncture \& Electro-Therapeutics Research 37(2-3): 89.

8. Wu P, Mills E, Moher D, Seely D (2010) Acupuncture in poststroke rehabilitation: a systematic review and meta-analysis of randomized trials. Stroke 41(4): e171.

9. Tao J, Zheng Y, Liu W, Yang S, Huang J, et al. (2015) Electro-acupuncture at LI 11 and ST 36 acupoints exerts neuroprotective effects via reactive astrocyte proliferation after ischemia and reperfusion injury in rats[J]. Brain Research Bulletin 120: 14-24.

10. Zhang JH, Wang D, Liu M (2014) Overview of Systematic Reviews and Meta-Analyses of Acupuncture for Stroke[J]. Neuroepidemiology 42(1): 50-58.

11. Winstein CJ, Stein J, Arena R, Bates B, Cherney LR, et al. (2016) Guidelines for Adult Stroke Rehabilitation and Recovery: A Guideline for Healthcare Professionals from the American Heart Association/American Stroke Association. Stroke 47(6): e98.

12. Gao Q Zhuo D (1994) A new assessment chart of motor function for CVA patients: development of simplified FMA. Chinese Journal of Rehabilitation Medicine.

13. Brunnstrom S (1966) Motor testing procedures in hemiplegia: based on sequential recovery stages. Physical Therapy 46(4): 357.

14. Lo YL, Cui SL (2003) Acupuncture and the modulation of cortical excitability. Neuroreport 14(9): 1229-1231.

15. Lo YL, Cui SL, Fook-Chong S (2005) The effect of acupuncture on motor cortex excitability and plasticity[J]. Neuroscience Letters 384(1-2): 145149.

16. Maioli C, Falciati L, Marangon M, Perini S, Losio A (2006) Short- and long-term modulation of upper limb motor-evoked potentials induced by acupuncture. European Journal of Neuroscience 23(7): 1931.

17. Zunhammer M, Eichhammer P, Franz J, Hajak G, Busch V (2012) Effects of acupuncture needle penetration on motor system excitability. Neurophysiologie Clinique/clinical Neurophysiology 42(4): 225-230.

18. Takeuchi N, Izumi S (2015) Combinations of stroke neurorehabilitation to facilitate motor recovery: perspectives on Hebbian plasticity and homeostatic metaplasticity. Frontiers in Human Neuroscience 9: 349.
ISSN: 2574-1241

DOI: $10.26717 /$ BJSTR.2020.25.004183

Li-dian Chen. Biomed J Sci \& Tech Res

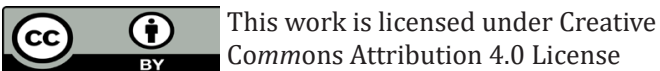

Submission Link: https://biomedres.us/submit-manuscript.php

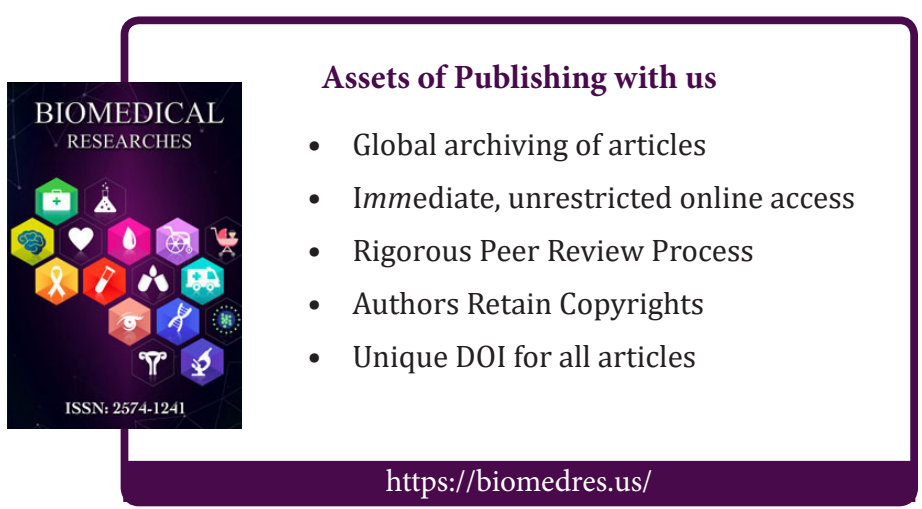

